\title{
Photoinduced Electron Transfer in a Radical SAM Enzyme Generates an S-Adenosylmethionine Derived Methyl Radical
}

Hao Yang ${ }^{1}$, Stella Impano ${ }^{2}$, Eric M. Shepard ${ }^{2}$, Christopher D. James ${ }^{1}$, William E. Broderick ${ }^{2}$, Joan B. Broderick ${ }^{2 *}$, Brian M. Hoffman ${ }^{1 *}$.

${ }^{1}$ Department of Chemistry, Northwestern University, Evanston, IL 60208. ${ }^{2}$ Department of Chemistry \& Biochemistry, Montana State University, Bozeman, MT. 59717. 


\section{Supplementary Text}

\section{$3 D$ vs $1 D$ rotational diffusion.}

The effects of rotation of the spectrum of $\cdot \mathrm{CH}_{3}$ are extremely complex, even ignoring, as appropriate in the present study, the possibility of quantum effects that are absent in HydG but, which appear in weakly interacting environments. This in part arises because of the unique set of hyperfine components of an $\alpha-{ }^{1} \mathrm{H}: \mathbf{A}\left({ }^{1} \mathrm{H}\right) \approx[-30,-90,-60] \mathrm{MHz}$, with the intermediate component, which equals $a_{i s o}\left({ }^{1} H\right)$, lying along the $2 \mathrm{p}_{z}$ orbital (along the normal to the $\mathrm{CH}_{3}$ plane), the small component lying along the $\mathrm{C}-\mathrm{H}$ bond, and the large component lying in the plane, normal to the bond. Rapid 3D diffusional rotation of course will average the anisotropic contribution, yielding the isotropic coupling of $\left|a_{i s o}\left({ }^{l} H\right)\right| \approx 60 \mathrm{MHz}$. However so will rapid $1 \mathrm{D}$ diffusional rotation about the normal, because the two in-plane components also average to $\left|a_{i s o}\left({ }^{1} H\right)\right|$.

The spectrum of ${ }^{13} \mathrm{CH}_{3}$ does distinguish between the two limiting rotational diffusion scenarios. A restriction to rapid 1D diffusion would not average the axial ${ }^{13} \mathrm{C}$ hyperfine tensor $\mathbf{A}\left({ }^{13} \mathrm{C}\right)=[-10,-10,260] \mathrm{MHz}$ given in the text, with its large component along the $2 \mathrm{p}_{\mathrm{z}}$ orbital, and the result for a frozen solution of ${ }^{13} \mathrm{CH}_{3}$ with z-axis fixed but rotation about $z$ would be a spectrum with isotropic couplings to the three ${ }^{1} \mathrm{H}$ but exhibiting the full anisotropic interaction with ${ }^{13} \mathrm{C}$ (Fig S4), contrary to observation. Thus although it is not likely that the limiting case of isotropic rotational diffusion actually obtains, nonetheless for our heuristic purposes it is appropriate to employ this limit to understand (see main text) how the tumbling of $\bullet \mathrm{CH}_{3}$ in the active site produces the observed spectrum, with isotropic couplings to the three ${ }^{1} \mathrm{H}$ and the ${ }^{13} \mathrm{C}$, but with enhanced linewidth in the ${ }^{13} \mathrm{CH}_{3}$ as a result of less-complete averaging of the ${ }^{13} \mathrm{C}$ hyperfine anisotropy, whose magnitude $\left(\left|\mathrm{A}_{1}-\mathrm{A}_{2}\right|\right)$ is $\sim 6.4$ times that of a proton. 


\section{Supplementary Figures}

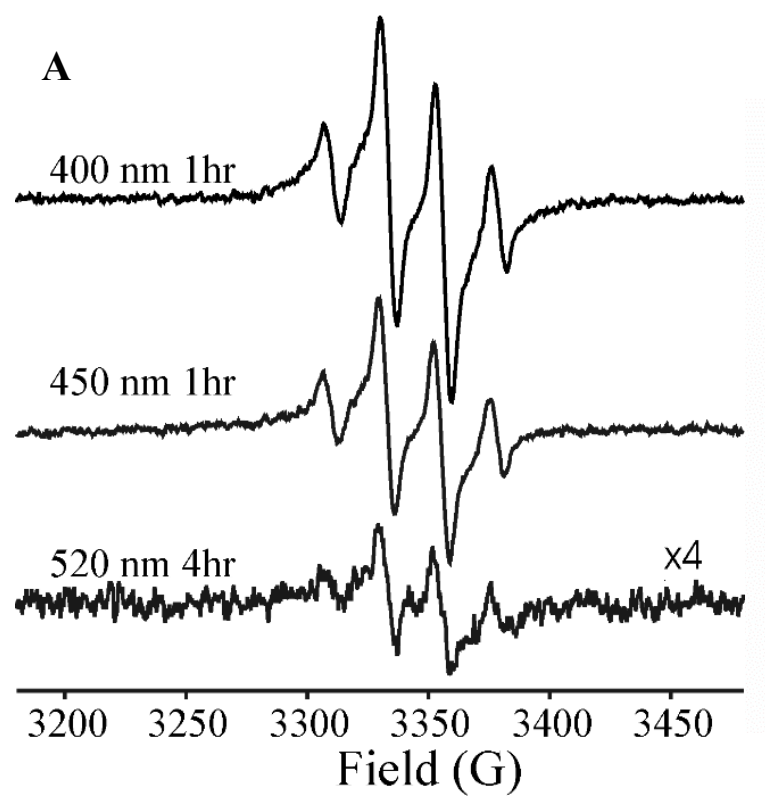

\section{B}

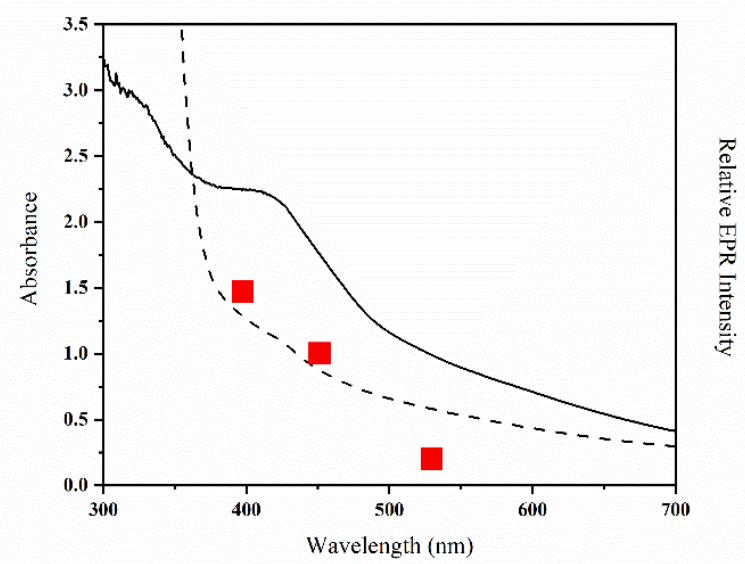

Figure S1. Wavelength dependence of photoinduced ET to generate the methyl radical.

A. X-band EPR spectra of photogenerated $\bullet \mathrm{CH}_{3}$ radical generated with the specified wavelength irradiation for the indicated amount of time. EPR Conditions: $\mathrm{T}=40 \mathrm{~K}$; modulation amplitude, $5 \mathrm{G}$; microwave frequency, $9.38 \mathrm{GHz}$. B. UV-visible spectra of asprepared (blue) and reduced (red) HydG, overlaid with the relative intensity of the methyl radical EPR signal (green squares) upon irradiation at the indicated wavelengths. 


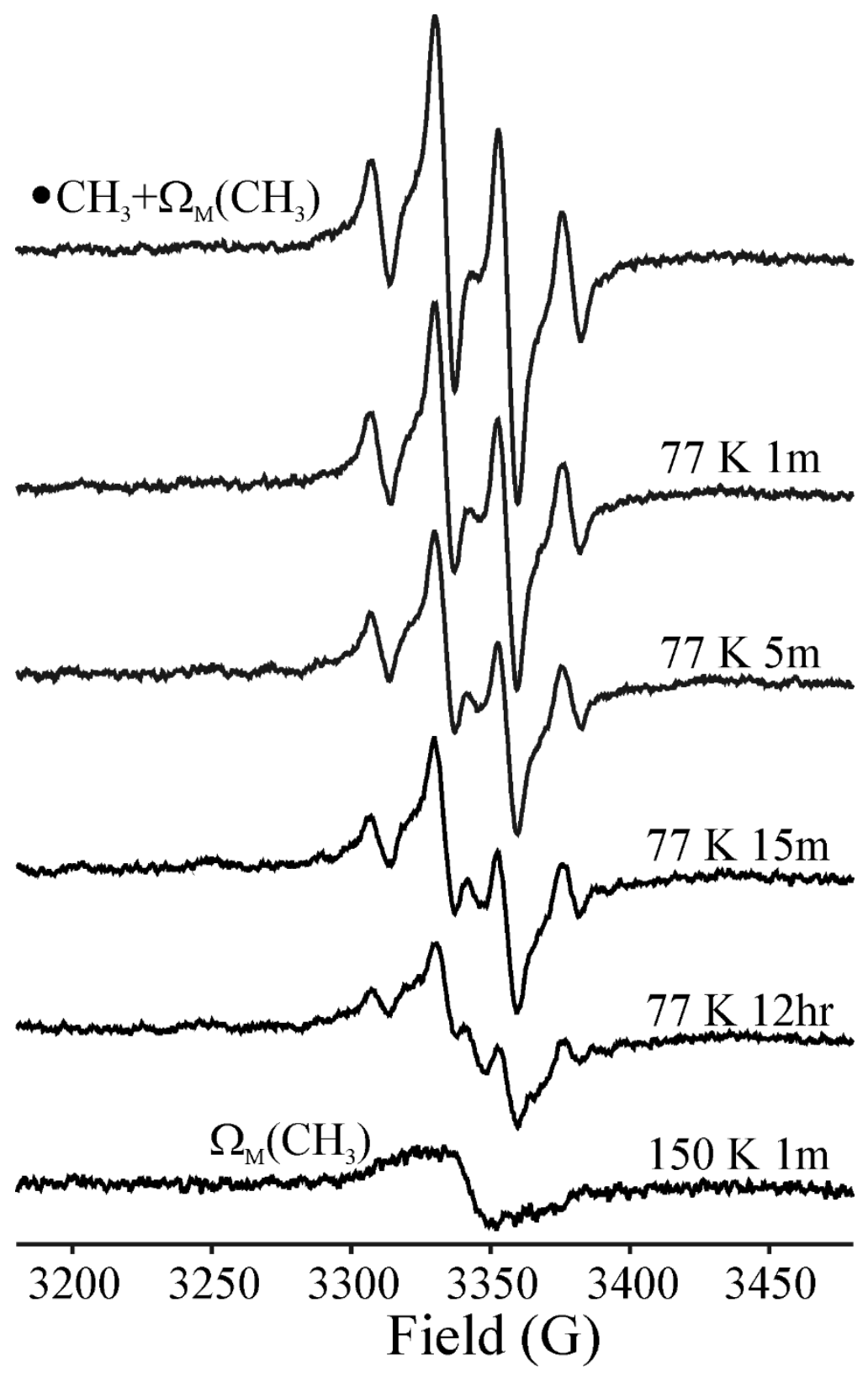

Figure S2. X-band EPR spectra of photogenerated - $\mathrm{CH}_{3}+\Omega_{\mathrm{M}}\left(\mathrm{CH}_{3}\right)$ annealed at indicated temperature. EPR Conditions: $\mathrm{T}=40 \mathrm{~K}$; modulation amplitude, $5 \mathrm{G}$; microwave frequency, $9.38 \mathrm{GHz}$. 


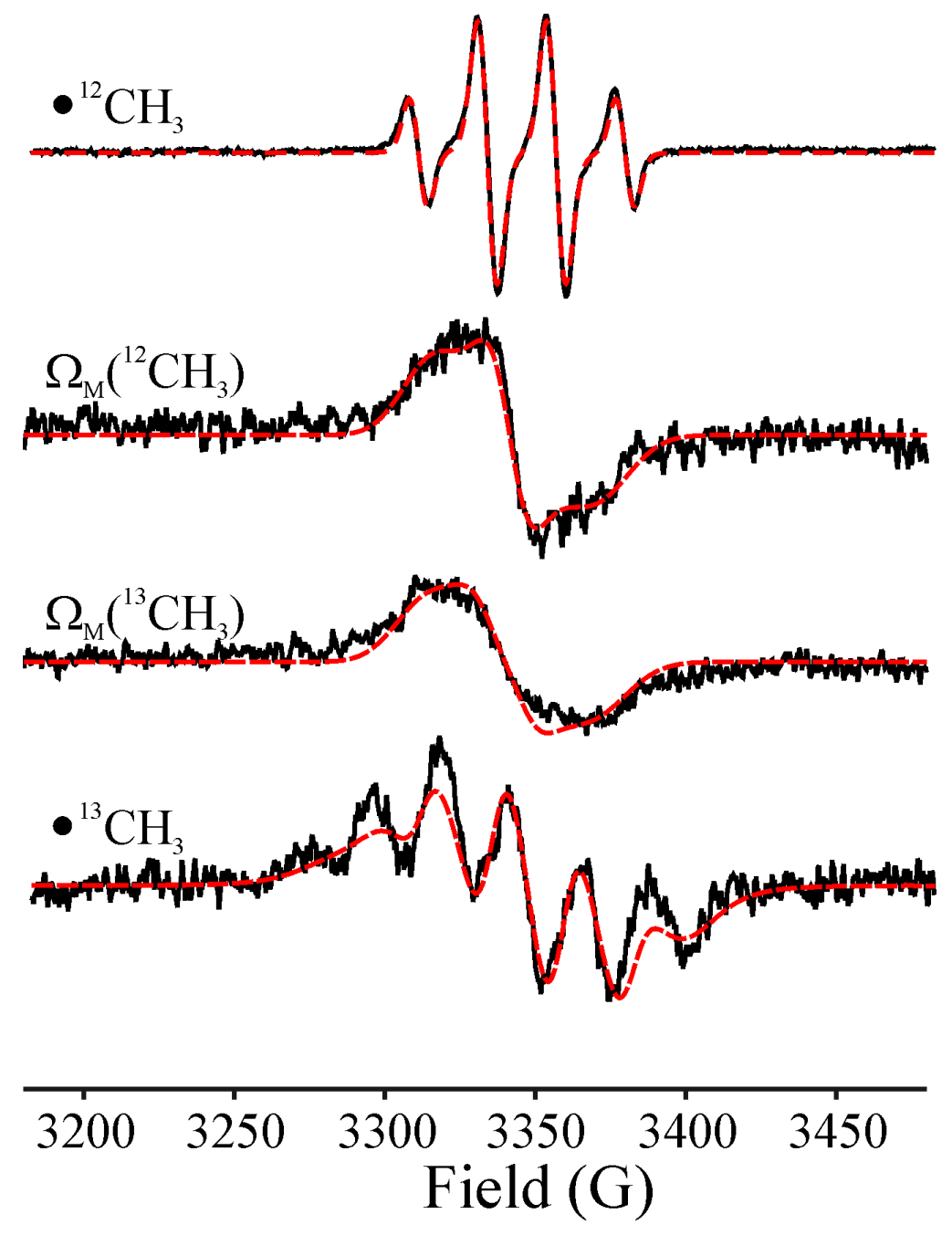

Figure S3. X-band EPR spectral comparison between $\bullet \mathrm{CH}_{3}$ $\left({ }^{12} \mathrm{C}\right.$ and $\left.{ }^{13} \mathrm{C}\right)$ and $\Omega_{\mathbf{M}}\left({ }^{12} \mathrm{C}\right.$ and $\left.{ }^{13} \mathrm{C}\right)$. The simulation (red) parameters are shown in the main text. EPR Conditions: $\mathrm{T}=$ $40 \mathrm{~K}$; modulation amplitude, $5 \mathrm{G}$; microwave frequency, $9.38 \mathrm{GHz}$. 


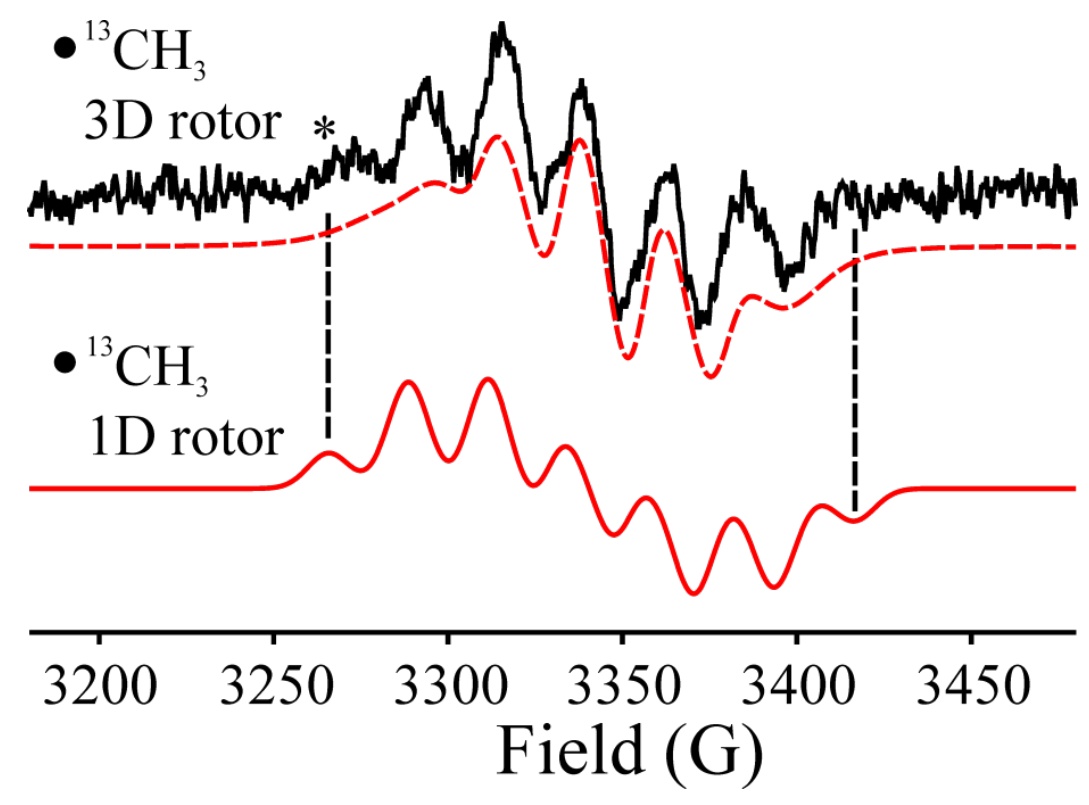

Figure S4. The $\mathrm{T}=40 \mathrm{~K}$ EPR spectrum of ${ }^{13} \mathrm{CH}_{3}$ and simulations for the models of a frozen solution of $3 \mathrm{D}$ and $1 \mathrm{D}$ rotational diffusion; $(*)$ represents small baseline 'flaw'. The EPR simulation parameters of the 3D rotor model is discussed in the main text. The EPR spectrum for the 1D rotor model is generated with parameters adapted from $5^{\prime}$-dAdo $\bullet: 3{ }^{1} \mathrm{H}$ with $\mathbf{A}_{\text {iso }}\left({ }^{1} \mathrm{H}\right)=64 \mathrm{MHz}, \mathrm{A}\left({ }^{13} \mathrm{C}\right)=[10,10,230] \mathrm{MHz}$ and $\mathrm{g}_{\text {iso }}=$ 2.004 . 


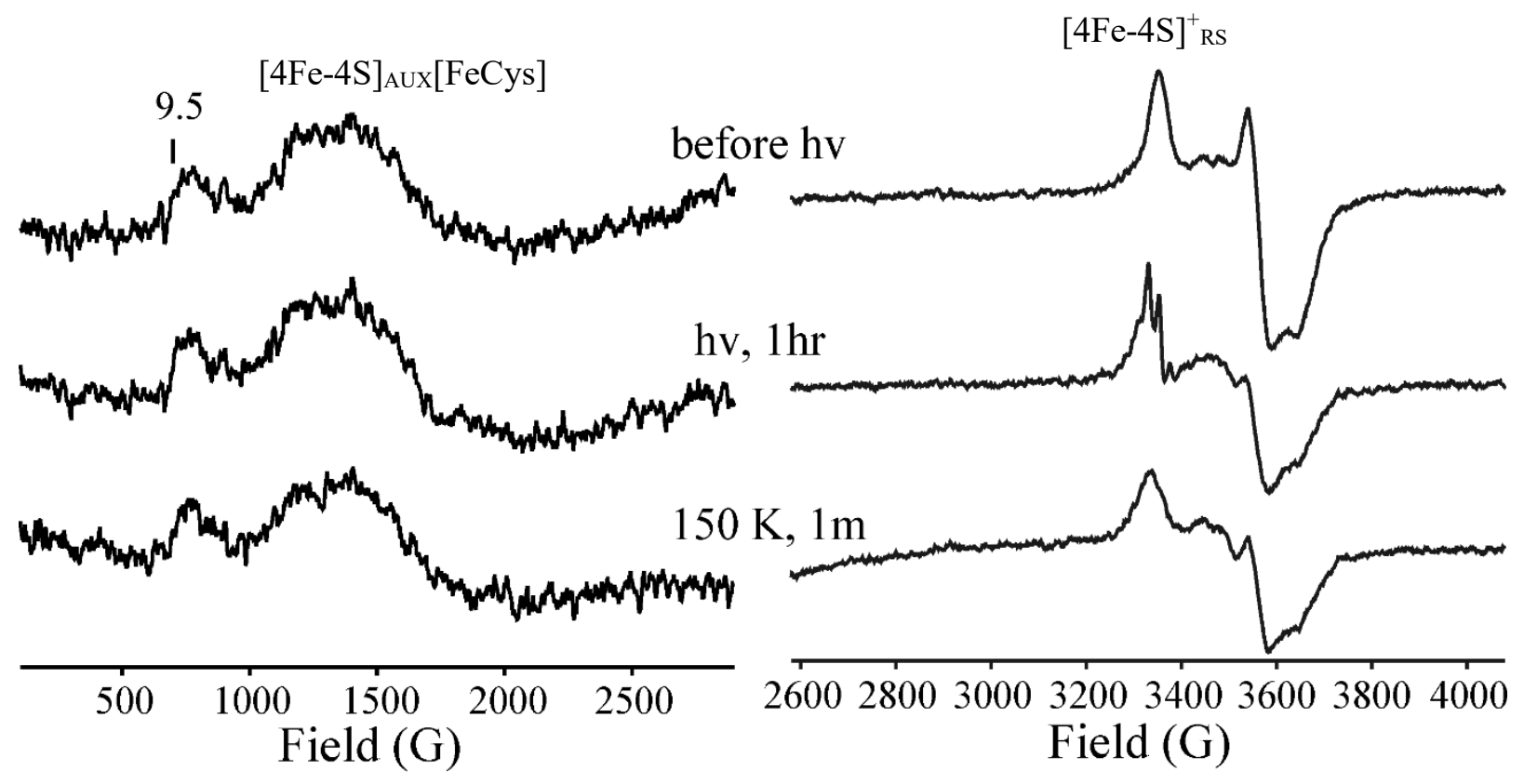

Figure S5. The X-band EPR spectra of $[4 \mathrm{Fe}-4 \mathrm{~S}]^{1+}$ Aux and $[4 \mathrm{Fe}-4 \mathrm{~S}]^{1+} \mathrm{RS}$ before and after photolysis, and after annealing $1 \mathrm{~m}$ at $150 \mathrm{~K}$. The $[4 \mathrm{Fe}-4 \mathrm{~S}]^{1+}{ }_{\text {Aux }}$ is associated with the characteristic $\mathrm{g}=9.5$ for $\mathrm{S}=5 / 2$, and the feature does not change after photolysis at $12 \mathrm{~K}$ and annealing at $150 \mathrm{~K}$. EPR conditions: $\mathrm{T}=12 \mathrm{~K}$; modulation amplitude, $5 \mathrm{G}$; microwave frequency, $9.38 \mathrm{GHz}$. 

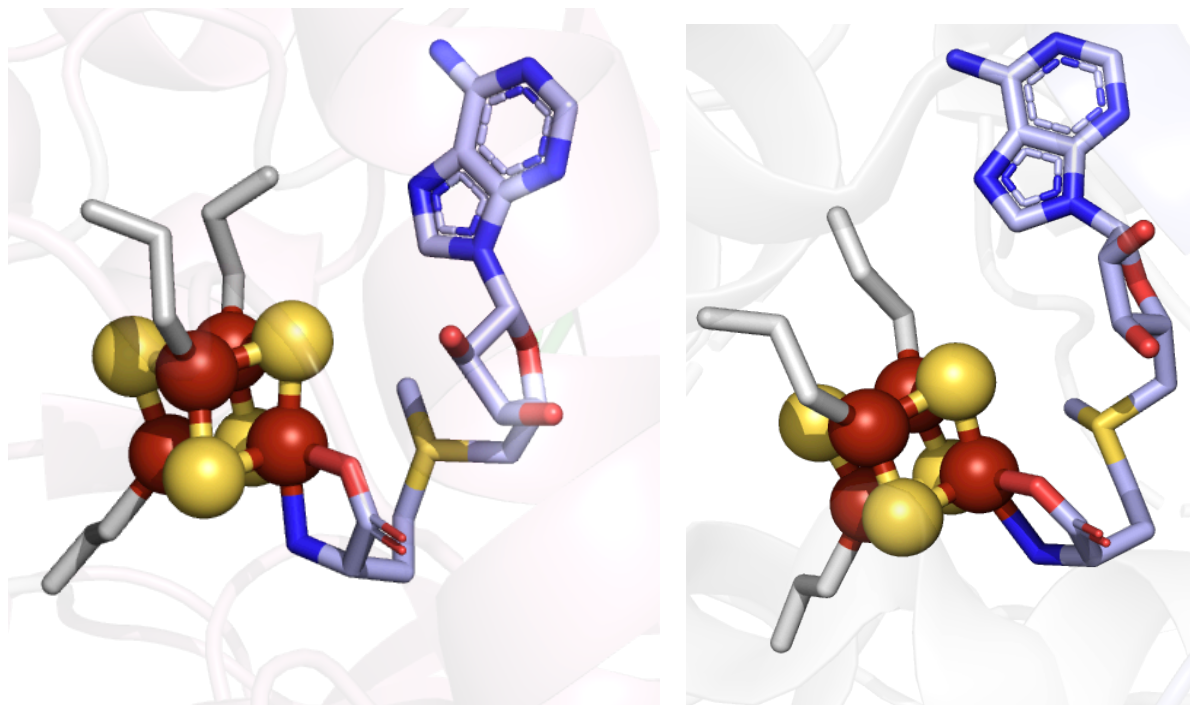

Figure S6. Comparison of the SAM-[4Fe-4S] cluster interaction in PFL-AE (left, 3CB8) and HydG (right, 4WCX). In both structures, the methionine moiety of SAM chelates the unique iron of the [4Fe-4S] cluster, and the S-C5' bond of SAM points away from the $[4 \mathrm{Fe}-4 \mathrm{~S}]$ cluster. 